\title{
Optimal Switching in Coplanar Orbit Transfer ${ }^{1}$
}

\author{
NGUYeN X. VINH ${ }^{2}$ AND RoBert DUdLey CULP ${ }^{3}$ \\ Communicated by T. N. Edelbaum
}

\begin{abstract}
The set of all controls that satisfy the Weierstrass necessary condition for optimality in the problem of time-open, coplanar orbit transfer via impulses is presented, along with the switching relations that must be satisfied at a corner in an optimal trajectory. This includes detailed data for eccentricities near unity. This study takes advantage of recently discovered closed-form solutions for the switching surfaces of this problem.
\end{abstract}

\section{Introduction}

The recent formulation of closed-form expressions for the switching conditions (Refs. 1-2) has rendered complete the solution of time-open, optimal, coplanar orbit transfer under conditions of unlimited thrust. The advances in this problem were made by applying the Weierstrass necessary condition and the Weierstrass-Erdmann corner condition in order to eliminate all but a small portion of the possible controls as nonoptimal. The results of this procedure for orbits with eccentricity less than 0.925 were presented in Ref. 3, along with a discussion of the method.

This approach may be viewed in a number of ways. It is an application of the maximum principle in a step-by-step manner. It may be considered as the enforcement of the triangle inequality on the characteristic velocity metric in the state space of coplanar elliptical orbits. Thus, the metric is completed by the smallest possible convex hull. The developable surface that

\footnotetext{
${ }^{1}$ Paper received July 8, 1970. Portions of this work were supported by NASA Contract No. NASr-54(06) and by NASA Grant No. NGR-06-003-033.

${ }^{2}$ Associate Professor, Department of Aerospace Engineering, The University of Michigan, Ann Arbor, Michigan.

${ }^{3}$ Assistant Professor, Department of Aerospace Engineering Sciences, University of Colorado, Boulder, Colorado.
} 
forms this hull has generators which are the optimal switches sought. The previously published results (Ref. 3) did not include the region of eccentricities larger than 0.925 because the switches began to include triple-point switches there. The computation by means of tangent planes was much more complicated. Also, the interest in such a large eccentricity was not high at that time.

The complications in the computation for high eccentricity have been eliminated by the appearance of the closed-form solutions to the switching function. Interest in the large-eccentricity results has increased particularly because of the problem of transition through highly eccentric orbits from a hyperbolic arrival velocity. The previous results (Ref. 3 ) have proven valuable in constructing a near-optimal controller for terminal orbit tailoring starting from hyperbolic arrival for the Viking mission. This required the extension of the complete numerical data to high eccentricities.

\section{Orbit Transfer as a Control Problem}

In this orbit transfer problem, there are two control variables, one specifying the position of the vehicle on the orbit and the second specifying the direction of the velocity impulse. The position on the orbit is measured by the true anomaly $\theta$ counted positive in the direction of the velocity in the orbit. The direction of the impulse is given by the angle $\psi$ measured up from the local horizontal in the same manner as the flight path angle (Fig. 1). The conditions of optimality considered here are local conditions and depend only on the eccentricity and the control variables. The impulse magnitude enters only to determine the elements of the osculating orbit as the impulse is increased from zero to its final magnitude.

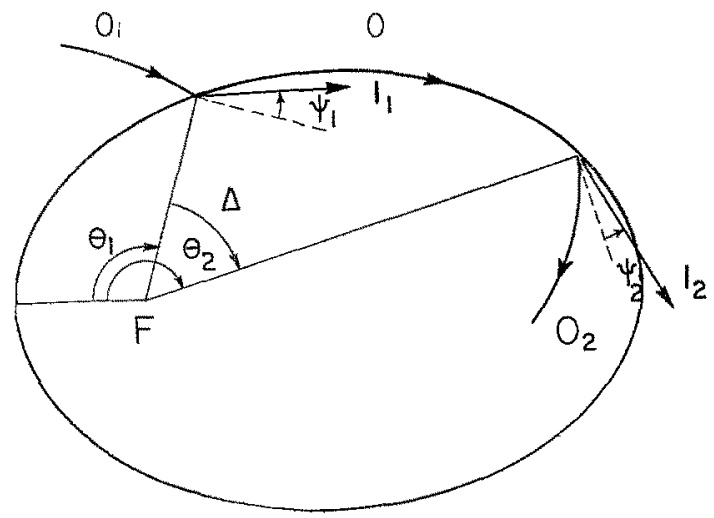

Fig. 1. Geometry of a switching. 
The controls that satisfy the necessary condition for optimality may be represented by regions in $e \theta \psi$-space. The dependency on the orbit appears only as the dependency on the eccentricity. For a given eccentricity, these regions of optimal controls represent the convex portions of the characteristic velocity metric. The boundary surfaces of these regions are the points at which an optimal control is discontinuous in $\theta$ and $\psi$. These switches (or corners) can be described by a mapping of the value of the true anomaly before and after the corner, $\theta_{1}$ and $\theta_{2}$. Since $e$ does not change during a switch, the surfaces in $e \theta \psi$-space provide the complete corner once $\theta_{1}$ and $\theta_{2}$ are known.

\section{Optimal Switching Relations in Closed Form}

The problem of determining the optimal trajectories thus leads to the key task of determining the switching surfaces in $e \theta \psi$-space along with the mapping of $\theta_{1}$ to $\theta_{2}$. While this can be handled indirectly, the discovery by Marchal (Ref. 2) of an exact relation among the control variables before a switch $\left(\theta_{1}\right.$ and $\left.\psi_{1}\right)$ and those after the switch $\left(\theta_{2}\right.$ and $\left.\psi_{2}\right)$ has made the computation much simpler. Marchal's relation is

$$
\cos \left(\psi_{1}+\psi_{2}\right)=\frac{\sin \left(\theta_{2}-\theta_{1}\right)-2 \cos \left(\theta_{2}-\theta_{1}\right) \tan \left(\psi_{2}-\psi_{1}\right)}{\sin \left(\theta_{2}-\theta_{1}\right)+\left[3+\cos \left(\theta_{2}-\theta_{1}\right)\right] \tan \left(\psi_{2}-\psi_{1}\right)}
$$

and is remarkable in that it is independent of the orbit's eccentricity.

Vinh (Ref. 1) has developed the exact relations for the optimal switching into such form as to make the computation purely routine. That paper includes a comprehensive discussion and tabulation of the switching relations to such an extent as to render redundant any further consideration of this problem. Vinh has supplied an exact expression for the eccentricity in terms of the control variables across a corner which makes possible the computation of the switching relations for all eccentricities, including $e$ near unity, in a straightforward manner. We have

$$
\begin{aligned}
& e^{2} \sin ^{2}(\Delta-\alpha)[1-\cos (\Delta-\alpha)][\sin (\Delta+\alpha)+3 \sin \alpha] \\
&=(1-\cos \alpha)[1-\cos (\Delta+\alpha)][2 \sin \Delta \cos \alpha+(3-\cos \Delta) \sin \alpha] \\
&+[1-\cos (\Delta-\alpha)][1-\cos \Delta \cos \alpha-2 \sin \Delta \sin \alpha] \\
& \quad \times\left[\sin \Delta \cos ^{2} \alpha+(3+\cos \Delta) \sin \alpha \cos \alpha-\sin \Delta \cos \alpha+2 \cos \Delta \sin \alpha\right] \\
&+3 \sin \alpha(1-\cos \Delta)[1-\cos (\Delta-\alpha)]\left[3(1+\cos \Delta) \cos ^{2} \alpha+4 \sin \Delta \sin \alpha \cos \alpha\right. \\
&\left.\quad-2 \cos \Delta \sin ^{2} \alpha\right]
\end{aligned}
$$


where

$$
\Delta=\theta_{2}-\theta_{1}, \quad \alpha=\psi_{2}-\psi_{1} .
$$

Finally, two expressions derived from the tangency requirements on the generators of the developable surface that covers the nonconvex portions of the characteristic velocity metric can be used to determine $\theta$ in terms of the other variables. We have

where

$$
A \sin \theta+B \cos \theta+C=0,
$$

$A \equiv e \cos \psi(\cos \psi-D), \quad B \equiv-e \sin \psi(\cos \psi-D), \quad C \equiv-\sin \psi(2 \cos \psi-D)$.

In the above relations, the control variables $\theta$ and $\psi$ are either all subscripted 1 or subscripted 2 and, when used for the first end of the switch,

$$
D_{1} \equiv\left[\sin \psi_{2}-\sin \psi_{1} \cos \left(\theta_{2}-\theta_{1}\right)\right] / \sin \left(\theta_{2}-\theta_{1}\right)
$$

and, for the second end,

$$
D_{2} \equiv\left[\sin \psi_{2} \cos \left(\theta_{2}-\theta_{1}\right)-\sin \psi_{1}\right] / \sin \left(\theta_{2}-\theta_{1}\right) .
$$

The equations of this section are sufficient to determine the control variables across a corner as a function of the orbit through the eccentricity.

\section{Results}

The controls that satisfy the necessary condition for optimality and relations for the controls that form an optimal corner are presented in Figs. 2-10. In all of these figures, upper signs go with upper signs, and lower signs go with lower signs. For a given eccentricity, the necessary condition is that the direction of the impulse must lie between the two bounds presented as the curves of $\psi$ versus $\theta$ in Figs. 2-3, (eccentricities less than 0.925). Figure 2 represents the maximum deviation from the horizontal which an optimal impulse may take. The dashed line is the locus of the Lawden points, and its equation is

$$
\tan \theta=4 n(2 n+1) \sqrt{ }\left(1-n^{2}\right) /(n-1)\left(8 n^{2}+5 n+1\right), \quad n=\cos 2 \psi .
$$

Figure 3 represents the minimum deviation. The dashed line is the locus of the symmetric points, and its equation is

$$
\tan 2 \psi=-2(\sin 2 \theta) /(3-\cos 2 \theta) .
$$




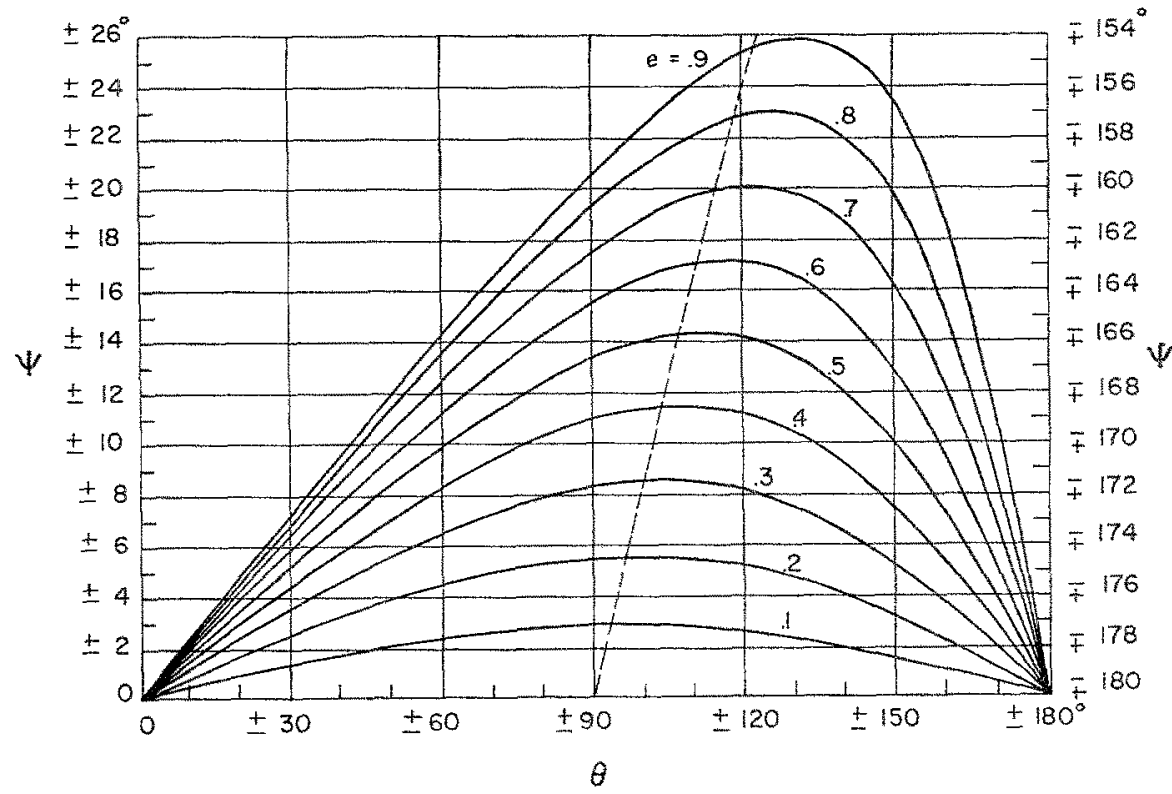

Fig. 2. Maximum deviation from the horizontal for the direction of an optimal impulse for eccentricities up to the eccentricity limit of 0.925 . The dashed line is the locus of the Lawden points.

Hence, the optimal thrust angle for symmetric switching passes through a maximum equal to

$$
\tan \psi= \pm(\sqrt{ } 3-\sqrt{ } 2), \quad \psi= \pm 17.6333^{\circ}, \quad \pm 162.3667^{\circ} .
$$

This corresponds to

$$
e=3(\sqrt{ } 6-1) / 5=0.86969, \quad \theta= \pm 144.7333^{\circ} .
$$

The switching relations are given in Figs. 4-5. Figure 4 represents the switches from one point on the surface of Fig. 2 to another. Figure 5 is the mapping for Fig. 3. These corners follow the general rule that the second impulse must yield a lower rate of increase in energy of the orbit than the first (Ref. 3).

These four figures correspond to the regular topology of the curves for the necessary condition as discussed in Ref. 1 . As the eccentricity approaches unity, the curves representing the upper and lower bounds on the angle of impulse intersect, producing an interval of $\theta$ on which no impulse is optimal. 


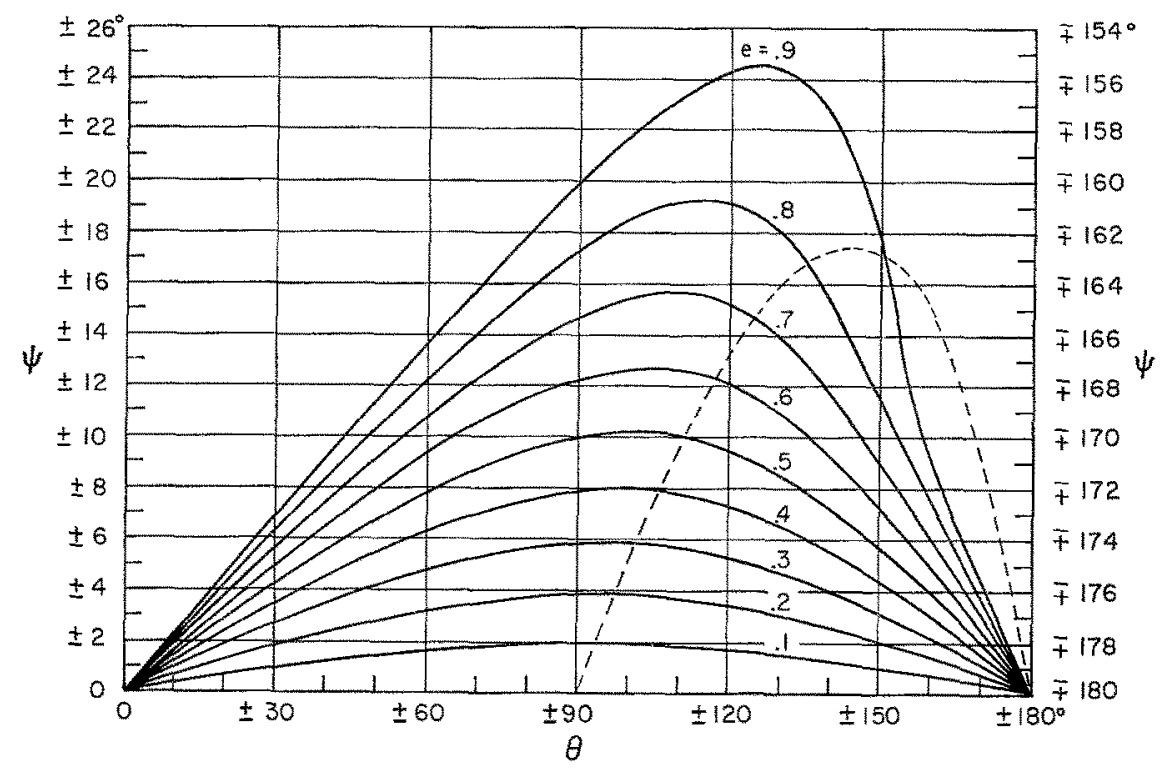

Fig. 3. Minimum deviation from the horizontal for the direction of an optimal impulse for eccentricities up to the eccentricity limit of 0.925 . The dashed line is the locus of the symmetric points.

The value of the eccentricity for which these curves first touch has become known as the eccentricity limit. Starting from this value, there exists a triple switching where the developable surface contains a plane. The values at this limit are

$$
\begin{aligned}
e & =0.92498988 \\
\theta_{1} & =\theta_{2}= \pm 122.3120845^{\circ} \\
\psi_{1} & =\psi_{2}= \pm 26.037475^{\circ} \text { or its supplement, } \\
\psi_{3} & = \pm 9.72974135^{\circ} \text { or its supplement, } \\
\theta_{3}-\theta_{1} & =77.754163^{\circ} .
\end{aligned}
$$

The curves representing the necessary condition and the switching relations for values of $e$ greater than 0.9 are given separately for $e=0.925$, $e=0.950$, and $e=0.975$ in Figs. 6-10. As can be seen, near periapsis, the necessary condition becomes essentially a line. The width between the upper and lower bounds is only a small fraction of a degree. In Fig. $6(e=0.925)$, the bounding curves are tangent at the Lawden point. For Figs. 7-8 $(e=0.950$ 


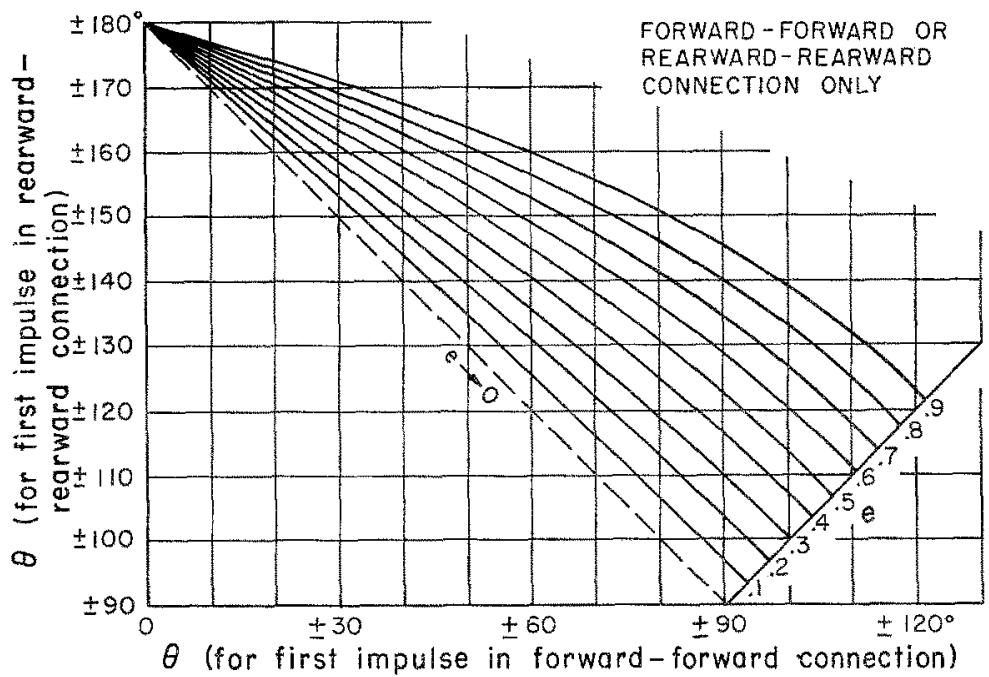

Fig. 4. Mapping between $\theta_{1}$ and $\theta_{\mathrm{g}}$, which represents the switching relations for the surface of Fig. 2. Switches are between two forward or between two rearward impulses only.

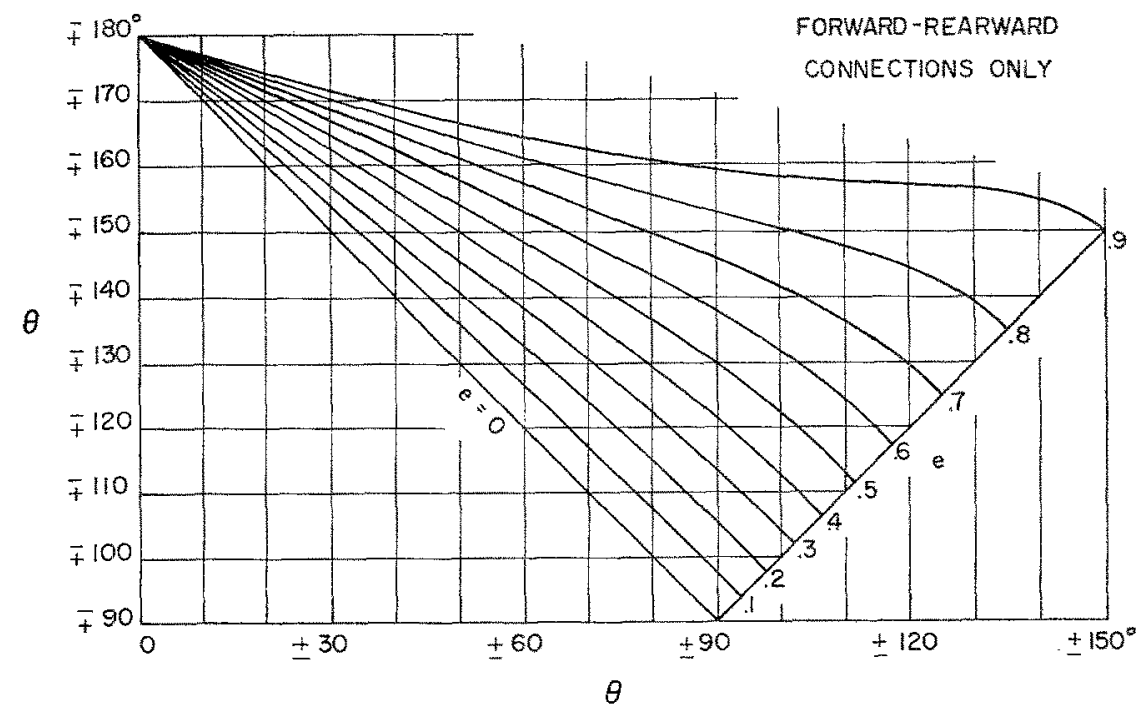

Fig. 5. Mapping between $\theta_{1}$ and $\theta_{2}$, which represents the switching relations for the surface of Fig. 3. Switches are between a forward first impulse and a rearward second impulse. 


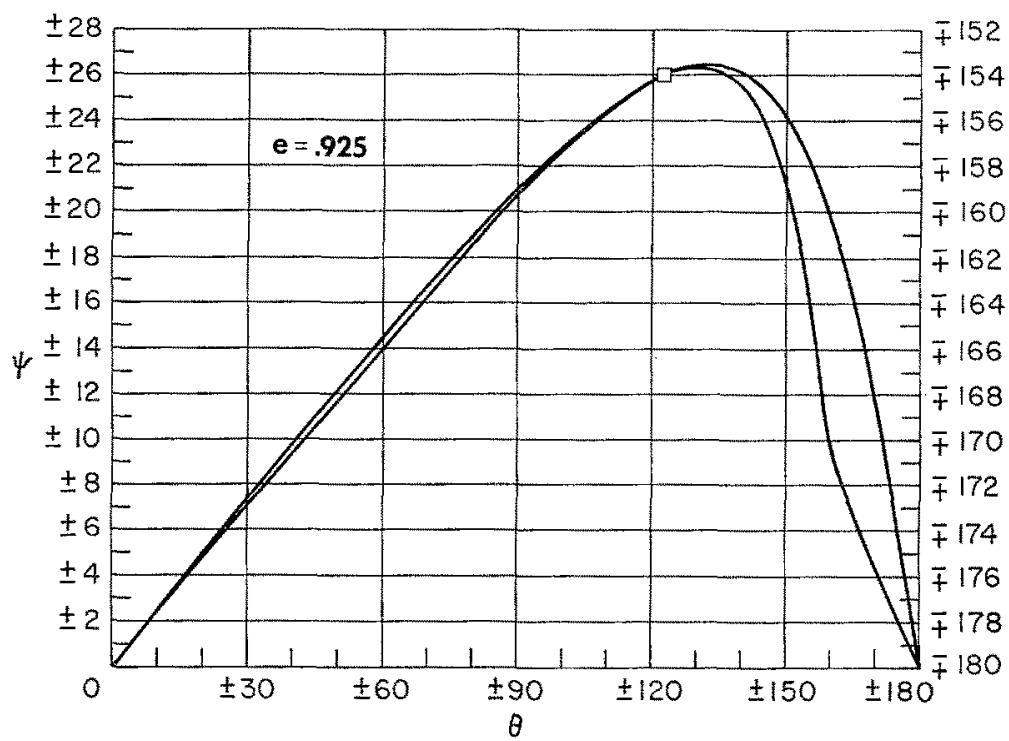

Fig. 6. Bounds on the optimal controls for $e=0.925$. The two bounds touch at the Lawden point.

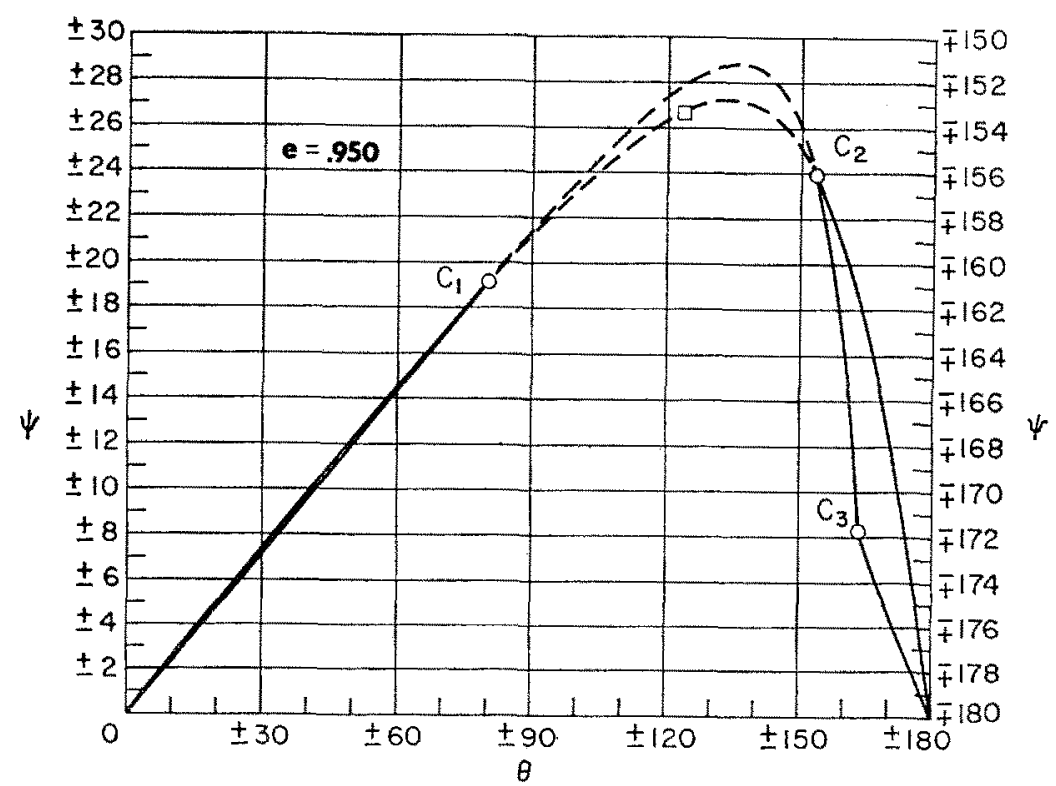

Fig. 7. Bounds on the optimal controls for $e=0.950$. The three circled points represent the triple switch condition. The dashed lines are the unsatisfiable bounds on the optimal controls. The Lawden point is indicated by the square. 


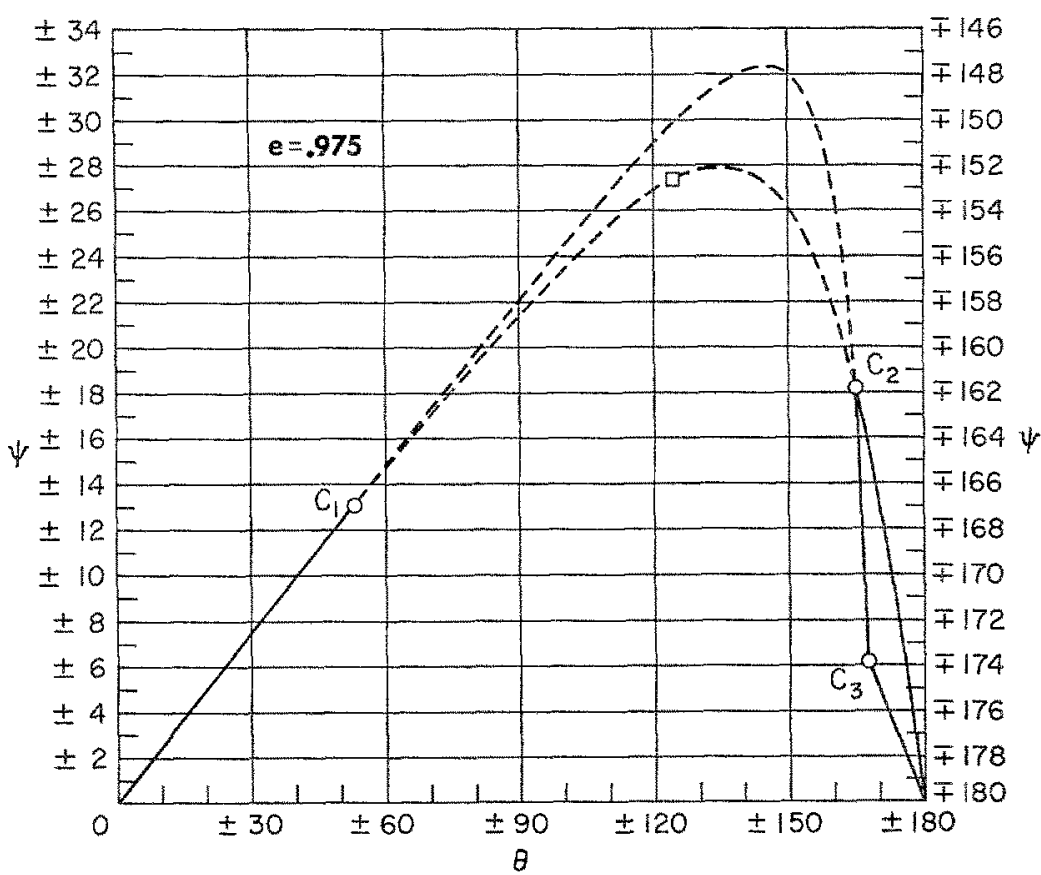

Fig. 8. Bounds on the optimal controls for $e=0.975$. The three circled points represent the triple switch condition. The dashed lines are the unsatisfiable bounds on the optimal controls. The Lawden point is indicated by the square.

and $e=0.975$ ), the three points circled in each figure represent the three simultaneous corner points discussed by Vinh (Ref, 1). The developable surface covering the metric in this region is formed by a triangle defined by these three points. The heavy lines in these figures represent the bounds where optimal impulses exist. The dashed lines represent the unsatisfiable bounds in the regions where the curves have crossed and no optimal impulse can exist. Figs, 9-10 give the switching relations for the upper and lower bounding curves, respectively, for these high eccentricities.

There exist several exact relations among the elements at the three simultaneous corner points. Let $C_{1}\left(\theta_{1}, \psi_{1}\right), C_{2}\left(\theta_{2}, \psi_{2}\right)$, and $C_{3}\left(\theta_{3}, \psi_{3}\right)$ be these points (Figs. 7-8). First, the general equations (1)-(6) are always valid for any switching pair, with the appropriate subscripts used. In addition, we have the following relations, which characterize the triple switching configuration:

$$
\cos \theta_{3}=\frac{\left(\sin ^{2} \psi_{1}+\sin ^{2} \psi_{2}\right) \cos \Delta-2 \sin \psi_{1} \sin \psi_{2}}{\left(\sin ^{2} \psi_{1}+\sin ^{2} \psi_{2}\right)-2 \sin \psi_{1} \sin \psi_{2} \cos \Delta}
$$




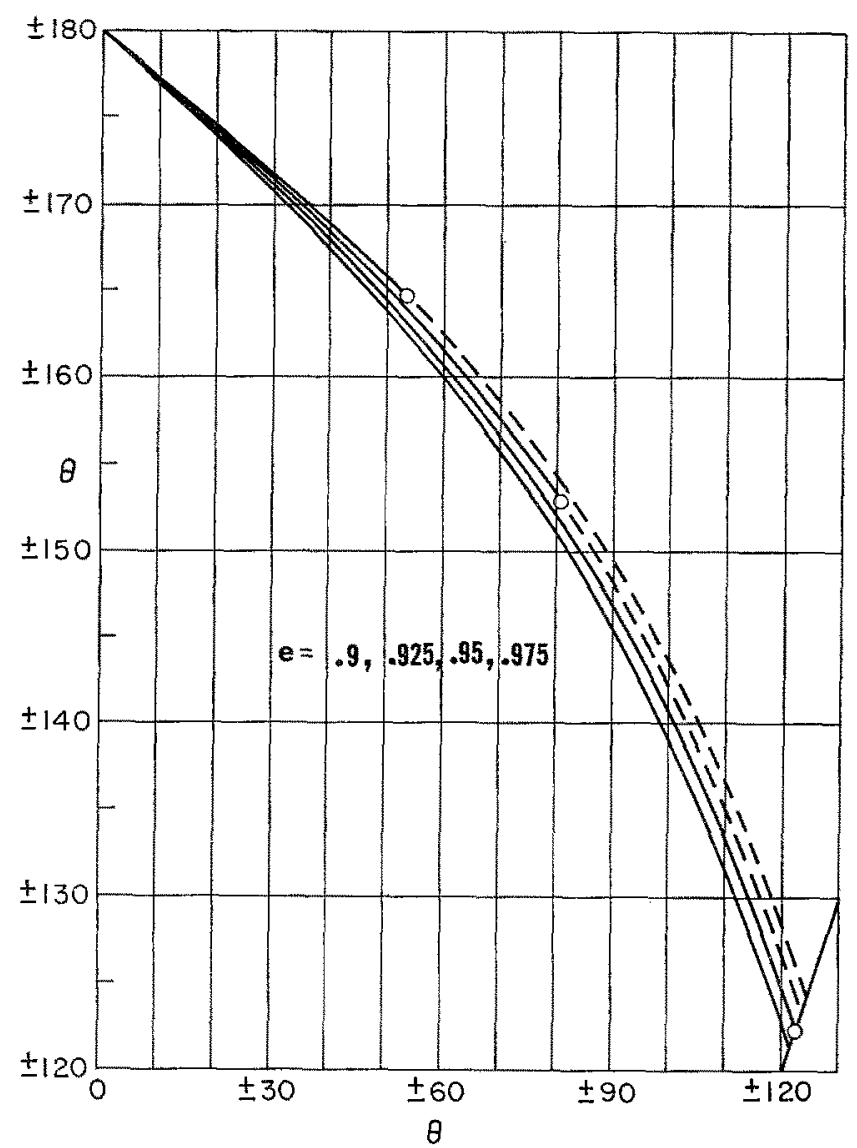

Fig. 9. Mapping between $\theta_{1}$ and $\theta_{2}$, which represents the switching relations for the upper curves in Figs. 6-8. Switches are between two forward or between two rearward impulses only.

or, in terms of $\alpha$ and $\Delta$,

$\cos \theta_{3}=\frac{\sin \alpha \cos \alpha \sin \Delta+(1+\cos \alpha)(1+2 \cos \alpha) \cos \Delta-3 \cos \alpha(1+\cos \alpha)}{\sin \alpha \cos \alpha \sin \Delta-(1+\cos \alpha)(2+\cos \alpha) \cos \Delta+3(1+\cos \alpha)}$.

Another relation is

$$
\tan \left(\frac{1}{2} \alpha\right) \tan \left(\frac{1}{2} \theta_{3}\right)+\tan \left(\frac{1}{2} \Delta\right) \tan \left[\frac{1}{2}\left(\psi_{2}+\psi_{1}\right)\right]=0 .
$$

For the third optimal thrust angle, we have

$$
\sin \psi_{3}=\left(\sin \theta_{1} \sin \psi_{1}-\sin \theta_{2} \sin \psi_{2}\right) / \sin \Delta .
$$




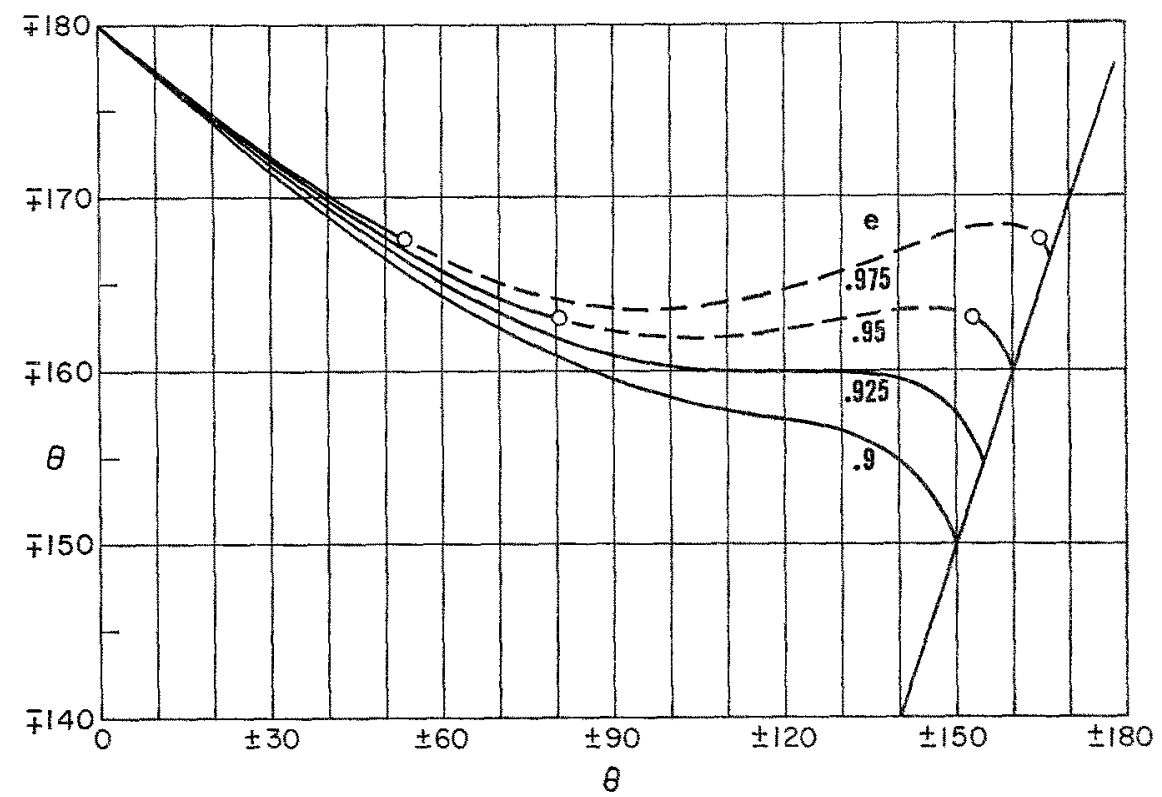

Fig. 10. Mapping between $\theta_{1}$ and $\theta_{2}$, which represents the switching relations for the lower curves in Figs. 6-8. Switches are between a forward first impulse and a rearward second impulse.

Also, we have

$$
\sin \psi_{2} / \sin \psi_{1}=\left[\sin \theta_{1}+\sin \left(\theta_{3}-\theta_{2}\right)\right] /\left[\sin \theta_{2}+\sin \left(\theta_{3}-\theta_{1}\right)\right] .
$$

All these relations are independent of the orbit eccentricity. They are in excess, but nevertheless useful because of their simplicity. When $\Delta$ tends to zero, the equations become trivial; but, using a limiting process to remove the singularity, we have, for the limiting case of Fig. 6 ,

$$
\tan ^{2}\left(\frac{1}{2} \theta_{3}\right)=4(2 n+1)^{2} /\left(1-n^{2}\right), \quad n=\cos 2 \psi_{1}=\cos 2 \psi_{2},
$$

where $n$ is given by

$$
1764 n^{6}+1890 n^{5}+207 n^{4}-572 n^{3}-309 n^{2}-60 n-4=0 .
$$

The angle $\theta_{1}=\theta_{2}$ is given by Eq. (7), and the eccentricity limit is obtained from

$$
e^{2}=9(1-n)\left(112 n^{4}+119 n^{3}+47 n^{2}+9 n+1\right) /(5 n+1)^{4}
$$


Finally, for third optimal thrust angle, we have

$$
\sin \psi_{3}= \pm 3(1-n)\left(6 n^{2}+3 n+1\right) \sqrt{ }[2(1-n)] / 2 e(5 n+1)^{2} .
$$

The most accurate set of values obtained to this date of this limiting case, as given by the relations above, were given in Eqs. (11). Extension of the Lawden singular point to the three-dimensional case has also been obtained recently (Ref. 4).

\section{Conclusions}

The recent presentation of closed-form solutions for the switching relations in the problem of time-open, optimal, coplanar orbit transfer via impulses has made feasible the display of complete results for the optimal controls and switching surfaces. In this paper, these results are presented graphically for all closed orbits, including the extension to orbits of eccentricity near unity. The results available previously had already proved valuable in providing a guide for construction of a near-optimal controller for orbit transfer, such as is required for the Viking mission. These results, along with the closed-form switching relations, should prove valuable in completing this construction.

Equivalent expressions for the three-dimensional orbit transfer problem are available (Ref. 2) and should lead to the completion of the general impulsive orbit transfer problem. This appears to be the one major problem left in this field.

\section{References}

1. VINH, N. X., Exact Relations of Optimum Sroitching in the Problem of Impulsive Transfer, Journal of the Astronautical Sciences, Vol. 17, No. 6, 1970.

2. Marchal, C., Transfers Optimaux entre Orbites Kepleriennes. Une Expression des Commutations sous Forme Finie, Astronautica Acta (to appear).

3. Culp, R. D., Contensou-Busemann Conditions for Optimal Coplanar Orbit Transfer, ATAA Journal, Vol. 5, No. 2, 1967.

4. Vinir, N. X., and Marchal, C., The Lawden's Singular Arcs in a Central Gravitational Field, Paper Presented at the 21st Congress of the International Astronautical Federation, Constance, West Germany, 1970. 